\begin{tabular}{c} 
International Journal of Advanced Astronomy, 4(2) (2016) 68-75 \\
International Journal of Advanced Astronomy \\
SPC \\
Website: $\begin{array}{c}\text { www. sciencepubco.com/index.php/IJAA } \\
\text { doi: } 10.14419 / i j a a . v 4 i 2.6227 \\
\text { Research paper }\end{array}$ \\
\hline
\end{tabular}

\title{
Resonance in the perturbations of a synchronous satellite due to angular rate of the earth-moon system around the sun and the earth's rotation rate
}

\author{
Sushil Yadav $^{1 *}$, Rajiv Aggarwal ${ }^{2}$, Bhavneet Kaur $^{3}$ \\ ${ }^{1}$ Department of Mathematics, Maharaja Agrasen College, University of Delhi, Vasundhara Enclave, Delhi-110096, India \\ 2 Department of Mathematics, Sri Aurobindo College, University of Delhi, Malviya Nagar, New Delhi-110017, India \\ ${ }^{3}$ Department of Mathematics, Lady Shri Ram College for Women, University of Delhi, Lajpat Nagar, Delhi-110024, India \\ *Corresponding author E-mail: syadav@mac.du.ac.in
}

\begin{abstract}
This paper investigates resonances in the perturbations of a synchronous satellite including its latitude, angular rate of the earth-moon system around the sun and the earth's rotation rate about its axis. This is found that resonances occur due to the commensurability between (i) angular velocity of the satellite and angular rate of earth's rotation about its axis and (ii) angular rate of the earth-moon system around the sun and angular rate of the rotation of the earth. Amplitude and time-period of the oscillation at the resonance points are determined using the procedure of Brown and Shook [3]. Effect of $\alpha$ (orbital angle of the mass-centre of the earth-moon system around the sun) on amplitude and time period is also analyzed. It is found that for increasing the values of $\alpha$ from $0^{\circ}$ to $45^{\circ}$ amplitude decreases and time period also decreases. Effect of time on the latitude of the satellite including earth oblateness is also studied. It is seen that for increasing the value of $t$, there is a small change in $\Delta \phi$, the latitude of the synchronous satellite.
\end{abstract}

Keywords: Earth Oblateness; Perturbations; Resonance; Synchronous Satellite.

\section{Introduction}

Resonances in a geosynchronous satellite are of great importance for its applications in telecommunication, navigation, mass-media, meteorological and geodetic studies. In this paper, we have considered the effect of the gravitational attraction of the sun and the moon. The ellipticity of the earth's equatorial section, the sun's radiation pressure and residual drag effects are neglected. We have investigated resonances in the perturbations of a synchronous satellite including its latitude, angular rate of the earth-moon system around the sun and rotation of the earth.

The phenomenon of resonance in the solar system is studied by many authors. Cook [4] studied the effects of the gravitational attractions of the Sun and Moon on the orbital elements of an Earth satellite using Lagrange's planetary equations. He identified fifteen resonance families and labeled them as numbers. Allan [1] investigated the motion in longitude of a nominally geostationary satellite due to the tesseral harmonics. He further developed the corrective impulses required etc., for the principal $\mathrm{J}_{2,2}$ term. Giacaglia [8] studied the double resonance on the motion of a satellite. Hagihara [10] studied resonance in a geocentric satellite due to the oblateness of the earth. The problem of resonance in Celestial Mechanics is surveyed by Garfinkel [7]. He formulated and studied the solution for the ideal resonance problem. Within the framework of the planar-elliptic R3BP, Wisdom [14] studied the long-term dynamics associated with the 3/1 resonance to study the chaotic zones as well as the zones of quasi-periodic librations in the 3/1 resonance. Hadjidemetriou [9] studied the relation between resonance and instability in the solar system and in the planetary system. Henrard [11] reviewed the study of the resonance cases $2 / 1$ and $3 / 1$ in the planar elliptic restricted three body problem. He showed how numerical experiments are significant in the celestial mechanics. Breiter [2] studied the eccentricity resonance between the secular motion of an Earth satellite's orbit and the longitudes of the Sun and the Moon within a Hamiltonian framework. Michtchenko et al. [12] studied a catalog of stable and unstable apsidal corotation resonance for the resonant planar planetary three body problem. Gallardo [6] studied the characteristics of the resonant disturbing function for an asteroid perturbed by a planet in circular orbit. He applied a numerical method to the region of the main belt of asteroids showing the relevance of several mean motion resonances (MMR) with several planets. Narayan and Singh [13] studied the existence of resonance and linear stability of the triangular equilibrium points of the planar restricted three body problem considering the photo gravitational effect of both the primaries in circular and elliptic case.

The paper organization is as follows:

Equations of motion of synchronous satellite are described in Sect. 2 while in Sect. 3 we have used the perturbation equations to express the equations of motion in the form of second order ordinary differential equations. From the solution of these equations, it is seen that the resonance occurs due to angular rate of rotation of the earth and angular rate of the earth-moon system around the sun. In Sect. 4 and 5, we investigated resonances due to earth's rotation rate and the angular rate of the earth-moon system around the sun. We applied a method as given in Brown and Shook [3] to determine amplitude and time period of the oscillation at the resonance point. Finally, Sect. 6 summarizes the conclusion of the paper. 


\section{Equations of motion}

The equations of motion of satellite $P(r, \theta, \phi)$ moving around the earth $E$ are given by (Frick and Garber [5])

$$
\begin{gathered}
\ddot{r}-r \dot{\theta}^{2}=-\frac{g_{0} R^{2}}{r^{2}}-\frac{3 J_{2} g_{0} R^{4}}{2 r^{4}} \\
+r \dot{\alpha}^{2}\left\{3(\cos \theta \cos \alpha+\sin \theta \sin \alpha \cos \varepsilon)^{2}-1\right\} \\
+\frac{r \dot{v}^{2}}{\mu}\left\{3(\cos \theta \cos v+\sin \theta \sin v \cos \varepsilon)^{2}-1\right\} \\
\frac{1}{r} \frac{d}{d t}\left(r^{2} \dot{\theta}\right)=3 r \dot{\alpha}^{2}(\cos \theta \cos \alpha+\sin \theta \sin \alpha \cos \varepsilon) \\
\cdot(-\sin \theta \cos \alpha+\cos \theta \sin \alpha \cos \varepsilon) \\
+\frac{3 r \dot{v}^{2}}{\mu}(\cos \theta \cos v+\sin \theta \sin v \cos \varepsilon) \\
(-\sin \theta \cos v+\cos \theta \sin v \cos \varepsilon), \\
\frac{1 d}{r \frac{d}{d t}\left(r^{2} \dot{\phi}\right)+r \dot{\theta}^{2} \phi}=-\frac{3 J_{2} g_{0} R^{4}}{r^{4} \phi} \\
-3 r \dot{\alpha}^{2}\left(\begin{array}{l}
\cos \theta \cos \alpha \\
+\sin \theta \sin \alpha \cos \varepsilon
\end{array}\right)(\sin \varepsilon \sin \alpha) \\
-\frac{3 r \dot{v}^{2}}{\mu}\left(\begin{array}{l}
\cos \theta \cos v \\
+\sin \theta \sin v \cos \varepsilon
\end{array}\right)(\sin \varepsilon \sin v)
\end{gathered}
$$

Where $g_{0}=$ gravitational aceleration on earth's surface,

$r=$ radial distance of the satellite $P$

from the centre of the earth $E$,

$\mathrm{J}_{2}=$ earth oblateness coefficient,

$\mu=$ mass ratio of the earth-moon system,

$\varepsilon=$ obliquity,

$\mathrm{R}=$ radius of the earth,

$\phi=\angle P E M^{\prime}=$ spherical coordinate of

satellite in a meridian plane, (Fig.1)

$\theta=\angle X " E M^{\prime}=$ spherical coordinate

of satellite in the equatorial plane,(Fig.1)

$\theta_{E}=\angle X " E F=$ angular position of the minor axis of the earth's equatorial section, (Fig.1)

$\dot{\theta}_{E}=$ angular rate of rotation of the earth, (Fig.2)

$\alpha=\angle X S G=$ orbital angle of mass-centre $G$ earth-moon system around the sun $S$,(Fig.1) $\dot{\alpha}=$ angular rate of mass-centre $G$ earth-moon system around the sun $S$,

$v=\angle \mathrm{X}^{\prime} \mathrm{GM}=$ orbital angle of earth-moon system about its centre of mass G, (Fig.1)

$\dot{v}=$ angular rate of earth-moon system about its centre of mass G,

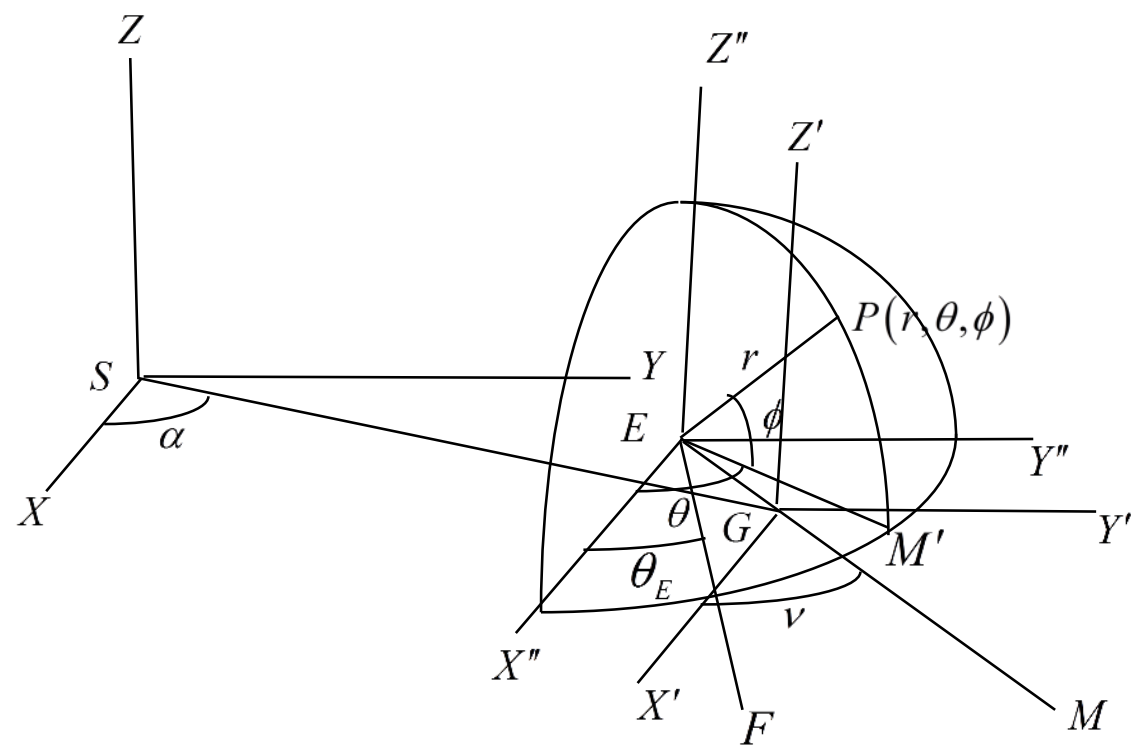

Fig. 1: Coordinate Systems 


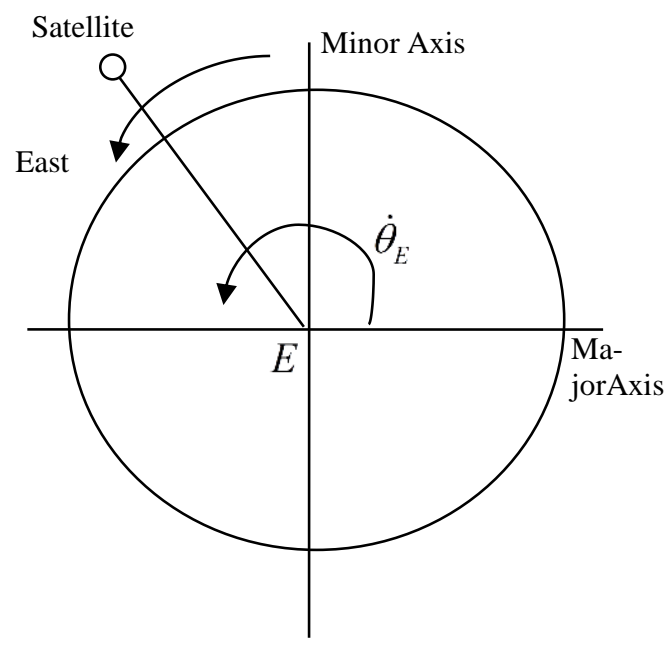

Fig. 2: Earth's Rotation Rate about Its Axis

$X, Y, Z=$ inertial coordinate system with origin at the centre of the sun

and the $X Y$ plane in the plane of ecliptic,

$X^{\prime}, Y^{\prime}, Z^{\prime}=$ inertial coordinate system with origin at the centre of the mass

of the earth-moon system and axis parallel to those of the $X Y Z$ system,

$X^{\prime \prime}, Y^{\prime \prime}, Z^{\prime \prime}=$ inertial coordinate system with origin at the centre of the earth

and axis parallel to those of the $X Y Z$ system,

\section{Perturbation equations}

We use a perturbation method in which the terms involving $\dot{\alpha}^{2}$ and $\dot{v}^{2}$ appear as time variable driving functions. The in-plane motion described by Equations (1) and (2) and the out-of-plane motion described by Equation (3) are uncoupled and can be dealt separately.

We define a set of perturbations relative to a synchronous orbit as follows:

$$
\mathrm{r}=\mathrm{r}_{\mathrm{c}}+\Delta \mathrm{r}
$$

$$
\dot{\theta}=\dot{\theta}_{\mathrm{E}}+\Delta \dot{\theta},
$$

Where $r_{c}$ is the radius of the synchronous orbit and $\dot{\theta}_{E}$ is the earth's angular rate about its axis.

By substituting the values of $r_{C}$ and $\dot{\theta}$ from Equations (4) and (5) into Equations (1) and (3) and following the procedure of Frick and Garber [5], we get the following perturbation equations for the in-plane motion and the out-of-plane motion relative to the synchronous equatorial orbit

$$
\begin{aligned}
\frac{d^{2} \Delta r}{d t}{ }^{2}+\dot{\theta}_{E}{ }^{2} \Delta r & =4 \dot{\theta}_{E}{ }^{2} \Delta r_{0}+\frac{3 r_{c} \dot{\alpha}^{2}}{4}\left\{-\frac{1}{3}+\cos ^{2} \varepsilon-\sin ^{2} \varepsilon \cos 2 \theta_{0}\right. \\
& -2 \sin ^{4} \frac{\varepsilon}{-} \cos 2\left(\theta_{0}+\alpha_{0}\right)-2 \cos ^{4} \frac{\varepsilon}{2} \cos 2\left(\theta_{0}-\alpha_{0}\right) \\
& +2 \sin ^{2} \varepsilon \cos 2 \theta+\sin ^{2} \varepsilon \cos 2 \alpha \\
& \left.+4 \sin ^{4} \frac{{ }^{4}}{2} \cos 2(\theta+\alpha)+4 \cos ^{4} \frac{\varepsilon}{2} \cos 2(\theta-\alpha)\right\}, \\
\frac{d^{2} \Delta \phi}{d t^{2}}+\dot{\theta}_{E}{ }^{2}\left(1+3 k_{1}\right) \Delta \phi & =-\frac{3 \sin \varepsilon}{2}\left(\dot{\alpha}^{2}+\dot{v}^{2}\right)\{\cos \varepsilon \sin \theta \\
& \left.+\sin ^{2} \frac{\varepsilon}{2} \sin (\theta+2 \alpha)-\cos ^{2} \frac{\varepsilon}{2} \sin (\theta-2 \alpha)\right\},
\end{aligned}
$$

Where

$$
k_{1}=J_{2} \frac{R^{2}}{r_{c}^{2}},
$$

$$
\dot{\theta}_{E}^{2}=\frac{g_{0} R^{2}}{r_{c}{ }^{3}},
$$

$$
\frac{\dot{\alpha}}{\dot{\theta}_{E}} \ll 1,
$$

$$
\begin{aligned}
\theta_{0} & =\text { initial value of } \theta, \\
\alpha_{0} & =\text { initial value of } \alpha, \\
\Delta r_{0} & =\text { intial value of } \Delta \mathrm{r}, \\
\Delta \phi & \approx \phi,(\phi \text { is regarded as a perturbation } \Delta \phi \text { in Equation (7)) } .
\end{aligned}
$$

We may take

$\theta=\dot{\theta} t, \alpha=\dot{\alpha} t$ and $v=\dot{v} t$.

Substituting these values in Equations (6) and (7), we get

$$
\begin{aligned}
\frac{d^{2} \Delta r}{d t}{ }^{2}+\dot{\theta}_{E}{ }^{2} \Delta r & =4 \dot{\theta}_{E}{ }^{2} \Delta r_{0}+\frac{3 r_{c} \dot{\alpha}^{2}}{4}\left\{-\frac{1}{3}+\cos ^{2} \varepsilon-\sin ^{2} \varepsilon \cos 2 \theta_{0}\right. \\
& -2 \sin ^{4} \frac{\varepsilon}{2} \cos 2\left(\theta_{0}+\alpha_{0}\right)-2 \cos ^{4} \frac{\varepsilon}{2} \cos 2\left(\theta_{0}-\alpha_{0}\right) \\
& +2 \sin ^{2} \varepsilon \cos 2 \dot{\theta} t+\sin ^{2} \varepsilon \cos 2 \dot{\alpha} t \\
& \left.+4 \sin ^{4} \frac{\varepsilon}{2} \cos 2(\dot{\theta}+\dot{\alpha}) t+4 \cos \frac{4}{2}-\cos 2(\dot{\theta}-\dot{\alpha}) t\right\}, \\
\frac{d^{2} \Delta \phi}{d t^{2}}+\dot{\theta}_{E}{ }^{2}\left(1+3 k_{1}\right) \Delta \phi & -\frac{3 \sin \varepsilon}{2}\left(\dot{\alpha}^{2}+\dot{v}^{2}\right)\{\cos \varepsilon \sin \dot{\theta} t \\
& \left.+\sin ^{2} \frac{\varepsilon}{2} \sin (\dot{\theta}+2 \dot{\alpha}) t-\cos ^{2} \frac{\varepsilon}{2} \sin (\dot{\theta}-2 \dot{\alpha}) t\right\} .
\end{aligned}
$$

Since we are interested in investigating the resonance in the motion of synchronous satellite due to $\dot{v}$, angular rate of the earthmoon system about its centre of mass and $\dot{\alpha}$, angular rate of the earth-moon system around the sun, we are ignoring the secular terms, we, therefore, write 


$$
\begin{aligned}
& \frac{d^{2} \Delta r}{d t^{2}}+\dot{\theta}_{E}{ }^{2} \Delta r=\frac{3 r_{c} \dot{\alpha}^{2}}{4}\left\{2 \sin ^{2} \varepsilon \cos 2 \dot{\theta} t+\sin ^{2} \varepsilon \cos 2 \dot{\alpha} t\right. \\
&\left.+4 \sin ^{4} \frac{\varepsilon}{2} \cos 2(\dot{\theta}+\dot{\alpha}) t+4 \cos ^{4} \frac{\varepsilon}{2} \cos 2(\dot{\theta}-\dot{\alpha}) t\right\} . \\
& \frac{d^{2} \Delta \phi}{d t^{2}}+\dot{\theta}_{E}{ }^{2}\left(1+3 k_{1}\right) \Delta \phi=-\frac{3 \sin \varepsilon}{2}\left(\dot{\alpha}^{2}+\dot{v}^{2}\right)\{\cos \varepsilon \sin \dot{\theta} t \\
&\left.+\sin ^{2} \frac{\varepsilon}{2} \sin (\dot{\theta}+2 \dot{\alpha}) t-\cos ^{2} \frac{\varepsilon}{2} \sin (\dot{\theta}-2 \dot{\alpha}) t\right\} .
\end{aligned}
$$

The solution of Equations (13) and (14) is given by

$$
\begin{aligned}
\Delta r & =A \cos (n t-B)+\frac{3 r_{c} \dot{\alpha}^{2}}{4}\left\{2 \sin ^{2} \varepsilon\left(\frac{1}{-4 \dot{\theta}^{2}+\dot{\theta}_{E}^{2}}\right) \cos 2 \dot{\theta} t\right. \\
& +\sin ^{2} \varepsilon\left(\frac{1}{-4 \dot{\alpha}^{2}+\dot{\theta}_{E}^{2}}\right) \cos 2 \dot{\alpha} t+4 \sin ^{4} \frac{\varepsilon}{2}\left(\frac{1}{-4(\dot{\theta}+\dot{\alpha})^{2}+\dot{\theta}_{E}^{2}}\right) \cos 2(\dot{\theta}+\dot{\alpha}) t \\
& \left.+4 \cos ^{4} \frac{\varepsilon}{2}\left(\frac{1}{-4(\dot{\theta}-\dot{\alpha})^{2}+\dot{\theta}_{E}^{2}}\right) \cos 2(\dot{\theta}-\dot{\alpha}) t\right\} \\
\Delta \phi & =C \cos \left(\sqrt{1+3 k_{1} \dot{\theta}_{E}}\right) t+D \sin \left(\sqrt{1+3 k_{1}} \dot{\theta}_{E}\right) t \\
& -\frac{3 \sin \varepsilon}{2}\left(\dot{\alpha}^{2}+\dot{v}^{2}\right)\left\{\cos \varepsilon\left(\frac{1}{-\dot{\theta}^{2}+\dot{\theta}_{E}^{2}\left(1+3 k_{1}\right)}\right) \sin \dot{\theta} t\right. \\
& +\sin ^{2} \frac{\varepsilon}{2}\left(\frac{1}{-(\dot{\theta}+2 \dot{\alpha})^{2}+\dot{\theta}_{E}^{2}\left(1+3 k_{1}\right)}\right) \sin (\dot{\theta}+2 \dot{\alpha}) t \\
& \left.-\cos ^{2} \frac{\varepsilon}{2}\left(\frac{1}{-(\dot{\theta}-2 \dot{\alpha})^{2}+\dot{\theta}_{E}^{2}\left(1+3 k_{1}\right)}\right) \sin (\dot{\theta}-2 \dot{\alpha}) t\right\}
\end{aligned}
$$

Where $A, B, C$ and $D$ are constants.

We know that if any one of the denominator vanishes, the motion is indeterminate at that point. From the solution (15), it is observed that resonance occurs at the points $\dot{\theta}=\frac{1}{2} \dot{\theta}_{E}$ and $\dot{\alpha}=\frac{1}{2} \dot{\theta}_{E}$ and from (16), we observed that resonance occurs at the point where $\dot{\theta}=\sqrt{1+3 k_{1}} \dot{\theta}_{E}$.

\section{Resonance due to angular rate of rotation of the earth}

To study the motion of satellite at the point where $\dot{\theta}=\sqrt{1+3 k_{1}} \dot{\theta}_{E}$ , we will follow the procedure as given in Brown and Shook [3]. It is proposed to find the solution of Equation (14) when that of the unperturbed equation

$$
\frac{d^{2} \Delta \phi}{d t^{2}}+\left(\dot{\theta}_{E}^{2} \sqrt{1+3 k_{1}}\right) \Delta \phi=0
$$

Is periodic and known.

The solution of Equation (17) is

$$
\Delta \phi=c \cos l,
$$

Where

$l=\left(\dot{\theta}_{E} \sqrt{1+3 k_{1}}\right) t+\varepsilon^{\prime}, \dot{\theta}_{E}=\frac{\sqrt{c_{1}}}{c \sqrt{1+3 k_{1}}}=$ function of $c$,

$$
\begin{aligned}
\frac{d^{2} \Delta \phi}{d t^{2}}+\left(\dot{\theta}_{E}^{2} \sqrt{1+3 k_{1}}\right) \Delta \phi & =-\frac{3 \sin \varepsilon}{2}\left(\dot{\alpha}^{2}+\dot{v}^{2}\right) \cos \varepsilon \sin \dot{\theta} t \\
& =-\frac{3}{4}\left(\dot{\alpha}^{2}+\dot{v}^{2}\right) \sin 2 \varepsilon \sin \dot{\theta} t \\
& =M A_{1} \sin n t \\
\frac{d^{2} \Delta \phi}{d t^{2}}+\left(m^{2} \sqrt{1+3 k_{1}}\right) \Delta \phi & =M \psi^{\prime} . \text { (say) }
\end{aligned}
$$

Where

$$
\begin{aligned}
& M=-\frac{3}{4}\left(\dot{\alpha}^{2}+\dot{v}^{2}\right), A_{1}=\sin 2 \varepsilon, m=\dot{\theta}_{E} \text { and } n=\dot{\theta}, \\
& \psi^{\prime}=\frac{\partial \psi}{\partial \Delta \phi}=A_{1} \sin n t, \psi=\Delta \phi A_{1} \sin n t, \\
& \psi=\frac{c}{2} A_{1}\{\sin (l+n t)-\sin (l-n t)\} .
\end{aligned}
$$

Then

$\frac{d c}{d t}=\frac{M}{K} \frac{\partial \Delta \phi}{\partial l} \psi^{\prime}=\frac{M}{K} \frac{\partial \psi}{\partial l}$,

$\frac{d l}{d t}=m \sqrt{1+3 k_{1}}-\frac{M}{K} \frac{\partial \Delta \phi}{\partial c} \psi^{\prime}=m \sqrt{1+3 k_{1}}-\frac{M}{K} \frac{\partial \psi}{\partial c}$,

Where

$$
\begin{aligned}
K & =\frac{\partial}{\partial c}\left(m \sqrt{1+3 k_{1}} \frac{\partial \Delta \phi}{\partial l}\right) \cdot \frac{\partial \Delta \phi}{\partial l}-m \sqrt{1+3 k_{1}} \frac{\partial^{2} \Delta \phi}{\partial l^{2}} \cdot \frac{\partial \Delta \phi}{\partial c}, \\
& =\text { a function of } c \text { only. }
\end{aligned}
$$

Since $m, K$ are functions of c only, we can put (20) and (21) into canonical form with new variables $c_{1}, B$ defined by

$d c_{1}=K d c$

$d B=-m \sqrt{1+3 K_{1}} d c_{1}=-m \sqrt{1+3 K_{1}} K d c$.

Equations (23) and (24) can be put in the form

$$
\begin{aligned}
& \frac{d c_{1}}{d t}=\frac{\partial}{\partial l}(B+M \psi), \\
& \frac{d l}{d t}=-\frac{\partial}{\partial c_{1}}(B+M \psi) .
\end{aligned}
$$

Differentiating Equation (21) with respect to $t$ and substituting the expressions for $\frac{\mathrm{dl}}{\mathrm{dt}}$ and $\frac{\mathrm{dc}}{\mathrm{dt}}$ in the result, we obtain

The arbitrary constants being $c$ and $\varepsilon^{\prime}$.

Equation (14), in our case, can be written as 


$$
\begin{aligned}
\frac{d^{2} l}{d t^{2}}= & \frac{M}{K}\left\{\frac{\partial\left(m \sqrt{1+3 k_{1}}\right)}{\partial c} \frac{\partial \psi}{\partial l}-m \sqrt{1+3 k_{1}} \frac{\partial^{2} \psi}{\partial l \partial c}-\frac{\partial^{2} \psi}{\partial c \partial t}\right\} \\
& +\frac{M^{2}}{K^{2}}\left\{\frac{\partial^{2} \psi}{\partial l \partial c} \frac{\partial \psi}{\partial c}-K \frac{\partial}{\partial c}\left(\frac{1}{K} \frac{\partial \psi}{\partial c}\right) \frac{\partial \psi}{\partial l}\right\} .
\end{aligned}
$$

Since the last expression of (25) has the factor $M^{2}$ it may, in general, be neglected in a first approximation.

In Equation (19), we find that $l$ and $t$ are present in $\psi$ as the sum of periodic terms with argument $l^{\prime}=l-n t$.

The affected term, in our case, is

$$
\psi=-\frac{1}{2} c A_{1} \sin l^{\prime}
$$

Equation (25) for $1^{\prime}$ is then

$$
\frac{d^{2} l^{\prime}}{d t^{2}}+\left(m \sqrt{1+3 k_{1}}-n\right)^{2} \frac{M}{K}\left\{\frac{\partial}{\partial c}\left(\frac{1}{\left(m \sqrt{1+3 k_{1}}-n\right)} \frac{\partial \psi}{\partial l^{\prime}}\right)\right\}=0,
$$

Or

$$
\frac{d^{2} l^{\prime}}{d t^{2}}-\left(m \sqrt{1+3 k_{1}}-n\right)^{2} \frac{M}{2 K}\left\{\frac{\partial}{\partial c}\left(\frac{c A_{1}}{m \sqrt{1+3 k_{1}}-n}\right)\right\} \cos l^{\prime}=0
$$

As a first approximation, we put

$c=c_{0}, m=m_{0}, K=K_{0}$ (all constants).

Then Equation (27) can be written as

$$
\frac{d^{2} l^{\prime}}{d t^{2}}-\left(m_{0} \sqrt{1+3 k_{1}}-n\right)^{2} \frac{M}{2 K_{0}}\left\{\frac{\partial}{\partial c}\left(\frac{c A_{1}}{m \sqrt{1+3 k_{1}}-n}\right)\right\} \cos l^{\prime}=0,
$$

where

$$
\begin{aligned}
K_{0} & =(K)_{0}, \\
& =\left\{\frac{\partial}{\partial c}\left(m \sqrt{1+3 k_{1}} \frac{\partial \Delta \phi}{\partial l}\right) \cdot \frac{\partial \Delta \phi}{\partial l}-m \sqrt{1+3 k_{1}} \frac{\partial^{2} \Delta \phi}{\partial l^{2}} \cdot \frac{\partial \Delta \phi}{\partial c}\right\}_{0} .
\end{aligned}
$$

Substituting $\Delta \phi=c \cos l \quad$ and and $l=m \sqrt{1+3 k_{1}} t+\varepsilon^{\prime},\left(m=\frac{\sqrt{c_{1}}}{c \sqrt{1+3 k_{1}}}\right)$, we obtain $K_{0}=\left\{\frac{\partial}{\partial c}\left(m \sqrt{1+3 k_{1}} \frac{\partial}{\partial l}(c \cos l)\right) \frac{\partial}{\partial l}(c \cos l)-m \sqrt{1+3 k_{1}} \frac{\partial^{2}}{\partial l^{2}}(c \cos l) \frac{\partial}{\partial c}(c \cos l)\right\}_{0}$, $=\left\{\sqrt{1+3 k_{1}} \frac{\partial}{\partial c}(-m c \sin l)(-c \sin l)-m \sqrt{1+3 k_{1}}\right.$, $\left.\frac{\partial}{\partial l}(-c \sin l)\left(\cos l+m \sqrt{1+3 k_{1}} t \sin l\right)\right\}_{0}$, $=\left\{\sqrt{1+3 k_{1}} c \sin l \frac{\partial}{\partial c}\left(\frac{\sqrt{c_{1}}}{\sqrt{1+3 k_{1}}} \sin l\right)\right.$ $\left.+\sqrt{1+3 k_{1}} m c \cos l\left(\cos l+m \sqrt{1+3 k_{1}} t \sin l\right)\right\}_{0}$, $=\left\{-m^{2} \sqrt{1+3 k_{1}} c t \sin l \cos l+m \sqrt{1+3 k_{1}} c \cos l(\cos l+m t \sin l)\right\}_{0}$,

$=\left\{\sqrt{c_{1}} \cos ^{2}\left(m \sqrt{1+3 k_{1}}+\varepsilon^{\prime}\right)\right\}_{0}$

$=\left\{\sqrt{c_{1}} \cos ^{2}\left(n t+\varepsilon^{\prime}\right)\right\}_{0}$

$=\sqrt{c_{1}} \cos ^{2}\left(\dot{\theta}_{t}+\varepsilon_{0}^{\prime}\right)=\sqrt{c_{1}} \cos ^{2}\left(\theta+\varepsilon_{0}^{\prime}\right)$

If the oscillation be small, then Equation (28) can be put in the form

$\frac{d^{2} l^{\prime}}{d t^{2}}-\left(m_{0} \sqrt{1+3 k_{1}}-n\right)^{2} \frac{M}{2 K_{0}}\left\{\frac{\partial}{\partial c}\left(\frac{c A_{1}}{m \sqrt{1+3 k_{1}}-n}\right)\right\}_{0}=0$,

Or

$\frac{d^{2} l^{\prime}}{d t^{2}}+\frac{M A_{1}}{2 K_{0}} c_{0}\left(\frac{\partial}{\partial c}\left(m \sqrt{1+3 k_{1}}\right)\right)_{0}=0$,

$\frac{d^{2} l^{\prime}}{d t^{2}}+\frac{M A_{1}}{2 K_{0}} c_{0}\left(\frac{\partial}{\partial c}\left(\frac{\sqrt{c_{1}}}{c}\right)\right)_{0}=0$,

$\frac{d^{2} l^{\prime}}{d t^{2}}-\frac{M A_{1}}{2 K_{0}} \frac{\sqrt{c_{1}}}{c_{0}}=0$,

$\frac{d^{2} l^{\prime}}{d t^{2}}=\frac{M A_{1}}{2 K_{0}} \frac{\sqrt{c_{1}}}{c_{0}}$.

Its solution is given by

$$
\begin{aligned}
& l^{\prime}=\frac{M A_{1}}{2 K_{0}} \frac{\sqrt{c_{1}}}{c_{0}} \frac{t^{2}}{2}+A t+B, \\
& l=\dot{\theta}_{t}+\frac{M A_{1}}{2 K_{0}} \frac{\sqrt{c_{1}}}{c_{0}} \frac{t^{2}}{2}+A t+B, \\
& l=\dot{\theta}_{t}-\frac{3}{16} \frac{\left(\dot{\alpha}^{2}+\dot{v}^{2}\right) \sin 2 \varepsilon}{c_{0} \cos ^{2}\left(\dot{\theta}_{t} t \varepsilon_{0}^{\prime}\right)} t^{2}+A t+B .
\end{aligned}
$$

Where A and B are constants of integration.

Using Equations (20) and (26), the equation for $c$ gives

$c=\frac{M A_{1}}{2 K_{0}} \frac{\sqrt{c_{1}}}{m n \sqrt{1+3 k_{1}}} \sin (l-n t)+d$,

$$
=-\frac{3}{8} \frac{\left(\dot{\alpha}^{2}+\dot{\nu}^{2}\right) \sin 2 \varepsilon}{\cos ^{2}\left(\dot{\theta} t+\varepsilon_{0}^{\prime}\right)} \frac{1}{m n \sqrt{1+3 k_{1}}} \sin (l-n t)+d .
$$

Where $\mathrm{d}$ is constant of integration and $l$ is determined from Equation (30).

Thus the perturbed solution is given by

$\Delta \phi=c \cos l$

Where the values $l$ of and ${ }^{c}$ are given by Equations (30) and (31) respectively.

We may choose the constants of integration

$A=0, B=0, d=0, c_{1}=1$ and $\varepsilon_{0}^{\prime}=0$

Thus we get 


$$
\begin{aligned}
\Delta \phi & =\left\{\frac{3}{8} \frac{\left(\dot{\alpha}^{2}+\dot{v}^{2}\right) \sin 2 \varepsilon}{\cos ^{2}\left(\sqrt{1+3 k_{1}} \dot{\theta}_{E}^{t}\right)} \frac{1}{\left(1+3 k_{1}\right) \dot{\theta}_{E}^{2}} \sin \left(\frac{3}{16} \frac{\sqrt{1+3 k_{1}} \dot{\theta}_{E}\left(\dot{\alpha}^{2}+\dot{v}^{2}\right) \sin 2 \varepsilon}{\cos ^{2}\left(\sqrt{1+3 k_{1}} \dot{\theta}_{E}^{t}\right)} t^{2}\right)\right\} \times \\
& \cos \left\{\sqrt{1+3 k_{1}} \dot{\theta}_{E} t-\frac{3}{16} \frac{\sqrt{1+3 k_{1}} \dot{\theta}_{E}\left(\dot{\alpha}^{2}+\dot{v}^{2}\right) \sin 2 \varepsilon}{\cos ^{2}\left(\sqrt{1+3 k_{1}} \dot{\theta}_{E} t\right)} t^{2}\right\} .
\end{aligned}
$$

To draw $\Delta \phi$ (latitude of satellite) versus time $t$, we use the following numerical values:

$$
\begin{aligned}
& R=6392.1 \times 10^{5} \mathrm{~cm}, \\
& r_{c}=42296 \times 10^{5} \mathrm{~cm}, \\
& J_{2}=1.08219 \times 10^{-3}, \\
& k_{1}=\frac{J_{2} R^{2}}{2}, \\
& \dot{\theta}_{E}=6.3004 \text { radians / solar day, } \\
& \dot{\alpha}=0.01720 \text { radians / solar day, } \\
& \dot{v}=0.22998 \text { radians / solar day, } \\
& \varepsilon=\left(23+\frac{27}{60}\right)^{\circ},
\end{aligned}
$$

We make the above quantities dimensionless by taking

$M_{E}+M_{S}=1$ unit

$D_{E S}=$ distance between the earth and the sun $=1$ unit,

$G=$ universal gravitational constant=1 unit.

Where

$M_{E}=$ mass of the earth=5.9742 $\times 10^{27} \mathrm{gm}$,

$M_{S}=$ mass of the sun=1.9891 $\times 10^{33} \mathrm{gm}$,

$D_{E S}=1.4959787061 \times 10^{13} \mathrm{~cm}$

$G=6.672 \times 10^{-8} \mathrm{~cm}^{3} \mathrm{gm}^{-1} \mathrm{sec}^{-2}$.

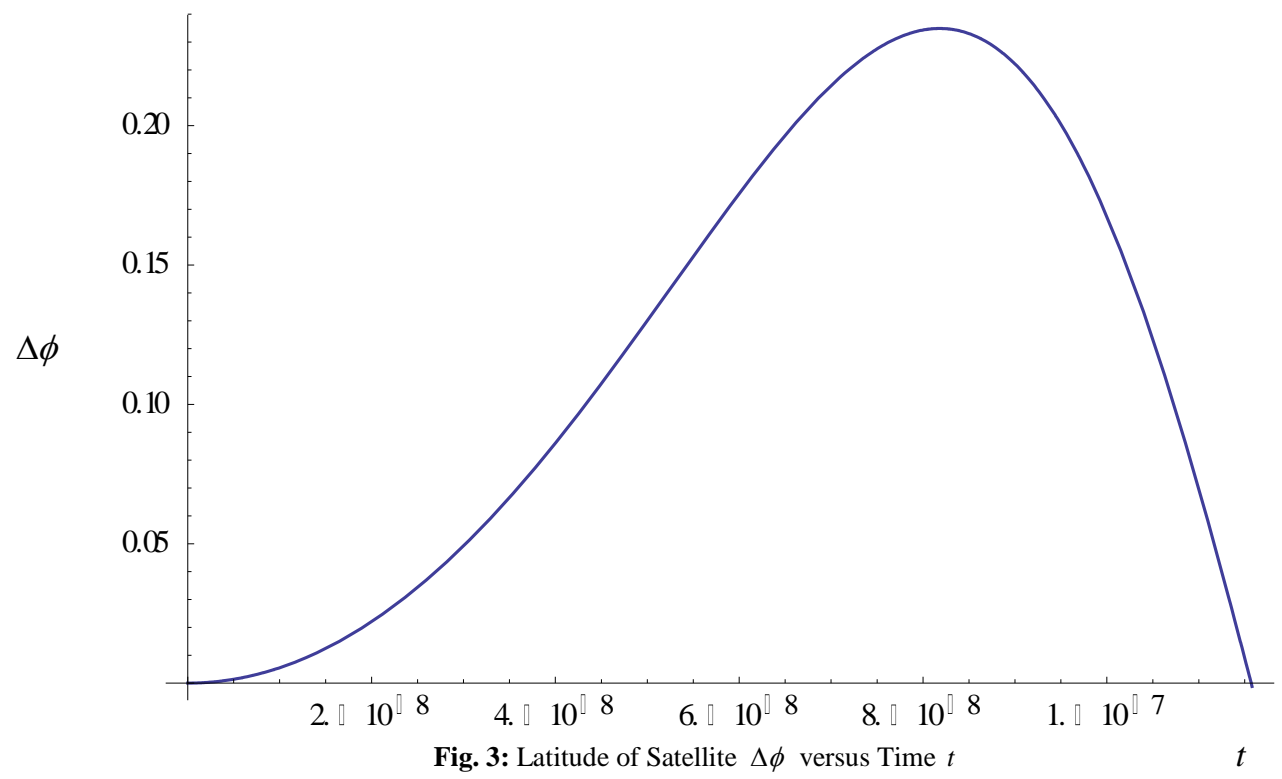

From Fig.3, we conclude that for increasing the value of $t$, there is a small change in $\Delta \phi$, the latitude of the synchronous satellite. The value of $\Delta \phi$ first increases and then decreases as time $t$ increases.

$$
A=\frac{2 \cos 2 \alpha}{\sqrt{3} \sqrt{r_{c}}(\dot{\alpha})^{\frac{3}{2}} \sin \varepsilon},
$$

\section{Resonance due to angular rate of the earth- moon system around the Sun}

Following the same procedure of Brown and Shook [3], we have determined the amplitude $A$ and time period $T$ of the resonance oscillation at the point where $\dot{\alpha}=\frac{1}{2} \dot{\theta}_{\mathrm{E}}$.

$$
\mathrm{T}=\frac{4 \pi \cos 2 \alpha}{\sqrt{3} \sqrt{\mathrm{r}_{\mathrm{c}}}(\dot{\alpha})^{\frac{3}{2}} \sin \varepsilon}
$$

We have drawn amplitude $A$ versus $\alpha$ (Fig.4) and time period $T$ versus $\alpha$ (Fig.5).

We observed that for increasing the values of $\alpha$ from $0^{\circ}$ to $45^{\circ}$, amplitude A decreases and time period $T$ also decreases. 

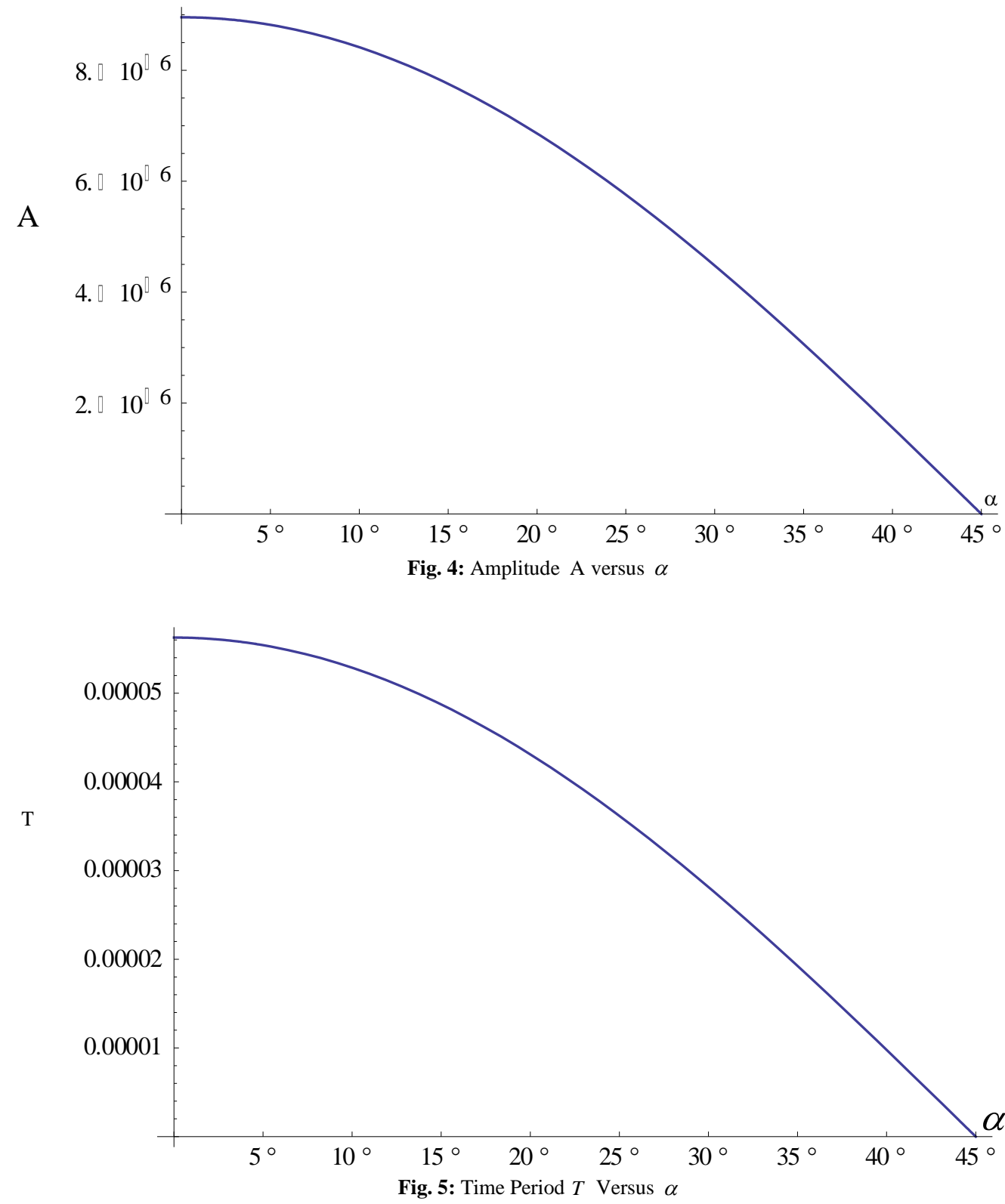

\section{Conclusion}

In this paper, we have studied resonances in the perturbations of a synchronous satellite resulting from the commensurability between (i) $\dot{\theta}$, angular velocity of the satellite and $\dot{\theta}_{E}$, angular rate of earth's rotation about its axis and (ii) $\dot{\alpha}$, angular rate of the earth-moon system around the sun and $\dot{\theta}_{E}$. We have taken into consideration the effects of the gravitational attraction of the sun and moon and the earth oblateness. The ellipticity of the earth's equatorial section, the sun's radiation pressure and residual drag effects are neglected. First, we have described the equations of motion of synchronous satellite by following the procedure of Frick and Garber [1962]. By using the perturbation equations (4) and (5), we have expressed the equations of motion in the form of second order ordinary differential equations (13) and (14). From the solutions (15) and (16), it is seen that the denominators vanishes at the points where $\dot{\theta}=\sqrt{1+3 k_{1}} \dot{\theta}_{E} \quad$ and $2 \dot{\alpha}=\dot{\theta}_{E}$ and the motion becomes indeterminate at these points. We applied a method given in Brown and Shook (1933) to study the motion at these resonance points. We have expressed $\Delta \phi$, the latitude of satellite in terms of time $t$ (Equation (32)). We have shown the effect of time $t$ on $\Delta \phi$ (latitude of the satellite). It is observed that for increasing the value of $t$, there is a little change in $\Delta \phi$, the latitude of the synchronous satellite (Fig.3). Finally, we have determined amplitude and time period of the oscillation at the resonance point $2 \dot{\alpha}=\dot{\theta}_{E}$. Using the data of a synchronous satellite, we have shown the effect of $\alpha$ (orbital angle of the earthmoon system around the sun) on amplitude and time period of the oscillation. We observed that for increasing the values of $\alpha$ from $0^{\circ}$ to $45^{\circ}$, amplitude $A$ decreases (Fig.4) and time period $T$ also decreases (Fig.5).

Acknowledgement we are thankful to the Centre for Fundamental Research in Space Dynamics and Celestial Mechanics (CFRSC), Delhi, India for providing all facilities for this research work.

\section{References}

[1] Allan RR (1963) Perturbations of a geostationary satellite by the longitude-dependent terms in the Earth's gravitational field. Planet Space Science, 11, 1325-1334.

[2] Breiter S (1999) Lunisolar apsidal resonances at low satellite orbits. Celestial Mechanics and Dynamical Astronomy, 74, 253-274. http://dx.doi.org/10.1023/A:1008379908163.

[3] Brown EW \& Shook CA (1933) planetary theory, Cambridge University Press, London and New York. Reprinted by Dover, New York. 
[4] Cook GE (1962) Luni-solar Perturbations of the orbit of an earth satellite. The Geophysical Journal of the Royal Astronomical Society, 6 (3), 271-291. http://dx.doi.org/10.1111/j.1365246X.1962.tb00351.x.

[5] Frick R H \& Garber T B (1962) Perturbations of a synchronous satellite. The RAND Corporation, R-399, NASA.

[6] Gallardo $T$ (2008) Evaluating the signatures of the mean motion resonances in the solar system. Journal of Aerospace Engineering, Sciences and Applications, vol.1, No.1.

[7] Garfinkel B (1982) On Resonance in celestial mechanics (A Survey). Celestial Mechanics, 28(1-2), 275-290. http://dx.doi.org/10.1007/BF01243738.

[8] Giacaglia G E O (1970) Double resonance in the motion of a satellite. Symposis Mathematics, 5, 45-63.

[9] Hadjidemetrio J D (1987) Resonances in the solar system and in planetary system. Proceedings $10^{\text {th }}$ ERAM of IAU, 3, 27-32.

[10] Hagihara Y (1972) Perturbation theory. Vol.II, Part-I, 462-469.

[11] Henrard J (1988) Resonances in the planar elliptic restricted body problem, in long term dynamical behavior of natural and artificial N body systems. (A. E. Roy ed.), NATO ASI series C-246, Kluwer Academic Publication, 405-425. http://dx.doi.org/10.1007/978-94009-3053-7 38.

[12] Michtchenko T A, Ferraz Mello S \& Beaugé, C (2006) Stationary orbits in resonant extra-solar planetary systems. Celestial Mechanics and Dynamical Astronomy, 94(4), 411-432. http://dx.doi.org/10.1007/s10569-006-9009-x.

[13] Narayan a \& Singh N (2014) Resonance stability of triangular equilibrium points in elliptical restricted three body problem under the radiating primaries. Astrophysics and Space Science, 353, 441-445. http://dx.doi.org/10.1007/s10509-014-2085-6.

[14] Wisdom J (1985) A perturbative treatment of the motion near the 3/1 commensurability. Icarus, 63, 279-282. 